\title{
Opportunistic Access of TV Spectrum Using Cognitive-Radio-Enabled Cellular Networks
}

\author{
Young-June Choi, Member, IEEE, and Kang G. Shin, Life Fellow, IEEE
}

\begin{abstract}
Motivated by the Federal Communications Commission's recent approval of commercial unlicensed operations of some television (TV) spectrum, we propose to integrate cognitive radios (CRs) that operate on unoccupied TV bands with an existing cellular network to increase bandwidth for mobile users. The existing cellular infrastructure is used to enable the operation of such CRs. Because base stations (BSs) can sense spectrum and exchange the sensed information for the reliable detection of primary users (PUs) and white spaces, we propose a collaborative sensing mechanism based on cell topology, where the BS declares its cell to be PU-free when neither the BS nor its neighboring BSs detect any PU. This way, in a PU-free cell, the following two types of channels are available: 1) channels that are originally licensed for the cellular system and 2) CR channels that are discovered through spectrum sensing. Because the CR channels that operate on TV bands usually suffer less path loss than the cellular channels, we derive two important results. First, each user gains more capacity when accessing a cellular channel than an empty TV channel, as long as intercell interferences are caused by the same sources. Second, assigning TV bands to cell-edge users is better in maximizing cell capacity. These two effects and the performance of the proposed sensing mechanism are verified through numerical evaluation.
\end{abstract}

Index Terms-Cellular networks, channel allocation, cognitive radios (CRs), television (TV) spectrum.

\section{INTRODUCTION}

$\mathbf{T}$ O PROVIDE higher throughput and enhanced quality of service, researchers have been exploring fourthgeneration $(4 \mathrm{G})$ wireless systems, which is also defined as International Mobile Telecommunications Advanced (IMTAdvanced). Recently, a significant amount of effort has been devoted worldwide to system evolution toward $4 \mathrm{G}$, such as the third-generation Long Term Evolution (3G LTE) and IEEE $802.16 \mathrm{~m}$ systems. However, the development of these nextgeneration wireless systems hinges on efficient solutions to the shortage of radio spectrum. Although the requirement on the supported data rates in IMT-Advanced is set to $100 \mathrm{Mb} / \mathrm{s}$

Manuscript received July 2, 2010; revised February 2, 2011 and June 13, 2011; accepted August 2, 2011. Date of publication August 12, 2011; date of current version October 20,2011. This work was supported in part by Ajou University through the new Faculty Research Fund and by the Ministry of Knowledge Economy, Korea, through the Information Technology Research Center Support Program, supervised by the National Information Technology Industry Promotion Agency (NIPA), under Grant NIPA-2011-(C1090-11210011). The review of this paper was coordinated by Dr. S. Wei.

Y.-J. Choi is with Ajou University, Suwon 443-749, Korea (e-mail: yjchoi@ ieee.org).

K. G. Shin is with the Department of Electrical Engineering and Computer Science, University of Michigan, Ann Arbor, MI 48109-2121 USA.

Color versions of one or more of the figures in this paper are available online at http://ieeexplore.ieee.org.

Digital Object Identifier 10.1109/TVT.2011.2164426 and $1 \mathrm{~Gb} / \mathrm{s}$ for high and low mobility, respectively, the total bandwidth of several hundred megahertz has been allocated for the use of IMT-Advanced [1], [2]. To achieve this required data rate, the following two approaches have been suggested: 1) Increase spectrum efficiency, and 2) make more radio spectrum available.

The latter approach is made feasible by the advent of the cognitive radio (CR) technology [3]. CRs allow secondary users (SUs) to access licensed bands that are unoccupied by licensed or primary users (PUs). Such a radio spectrum is found in television (TV) broadcast bands (e.g., $54-862 \mathrm{MHz}$ in North America) that possess good spectrum characteristics, e.g., wide transmission range. Recently, the U.S. Federal Communications Commission (FCC) has approved commercial unlicensed operations in the ultra high frequency (UHF) spectrum [4]. The possible use of the unused TV spectrum has led to creation of the CR-based standard IEEE 802.22 Wireless Regional Area Network (WRAN), which is a draft with a specified coverage of $33 \mathrm{~km} \mathrm{[5],} \mathrm{[6],} \mathrm{where} \mathrm{PUs} \mathrm{will} \mathrm{be} \mathrm{TV} \mathrm{boxes} \mathrm{and} \mathrm{a} \mathrm{TV} \mathrm{tower.}$

CRs can thus be used to make more radio spectrum resources available. Instead of independently operating an IEEE 802.22 network, a service provider is likely to jointly operate a CR network with an existing cellular system. Such a network overlay, called a CR-enabled cellular network, may be a promising business model for third-generation (3G) service providers, because they can offer more bandwidth to mobile end users by accessing more radio spectrum such as TV bands. ${ }^{1}$ In particular, the infrastructure that has been deployed for $3 \mathrm{G}$ services will facilitate the deployment of a CR system, e.g., how we can distribute spectrum sensors and collect the sensed data.

Already-installed base stations (BSs) can be used as the spectrum sensors and resource managers of CR networks. When information on PU locations and channel usage is provided to these cellular BSs through a shared database, BSs can easily access the unused channels as a secondary system without spectrum sensing. When such information is not provided, as assumed in this paper, spectrum sensing for the discovery of white spaces and PU signals is critical, because the CR system not only opportunistically accesses radio spectrum but is also required to quickly vacate the spectrum band whenever its PU returns. Because BSs are usually installed on a tall mast or the rooftop of a building, they can sense the presence of PUs very well. Considering that the transmission range of

\footnotetext{
${ }^{1}$ In practice, IEEE 802.16 and 802.22 systems are based on the common functionality of scalable OFDMA, which, together with the software-defined radio (SDR) technology, will enable such a network operation [7].
} 
cellular systems is typically several kilometers and the transmission range of TV broadcasting antennas is up to hundreds of kilometers, there could be dozens of cellular BSs in the coverage of each TV broadcasting antenna. Cellular BSs are also connected through a wired backhaul network so that the sensed information can quickly and reliably be exchanged among BSs through this backhaul network, thus requiring no additional radio resource for the dissemination of the sensed information. As a result, this approach will decrease the cost of operating spectrum sensors and collecting the sensed information. ${ }^{2}$

We propose a new collaborative sensing mechanism by augmenting already-deployed cellular BSs with CR capability. Using the sensed information from its neighboring BSs, each BS allows mobile terminals (MTs) ${ }^{3}$ within its cell to access a certain CR channel. When all the neighboring BSs do not detect any PU on the channel, the BS declares its cell area to be completely $P U$-free, because the possibility of interfering with any PU due to SU communications in the cell area is zero or very low. When a BS does not detect any PU but some of its neighboring BSs detect PUs on a specific CR channel, the BS declares its cell area to be partially PU-free, and MTs that are not close to BSs that report the presence of a PU are allowed to access the channel. To fulfill the partially PU-free option, each MT should report its discovery of BSs based on the pilot signals to its server BS. ${ }^{4}$

Because some BSs may provide MTs with more bandwidth through CR channels in a CR-enabled cellular network, the following two types of accessible channels are available: 1) channels that are originally licensed for the cellular system and 2) channels that are found through the CR system but are not used by their PUs. In general, CR channels that operate on TV bands have lower carrier frequency than cellular systems, thus suffering less path loss. Therefore, MTs will achieve higher capacity when accessing an idle TV channel for a single cell. However, in multicell environments, MTs achieve higher capacity by accessing the original licensed channel, instead of accessing an idle TV channel, when the intercell interference is generated by the same sources (i.e., neighboring cells). This case is our first finding on channel allocation. Another finding is that TV channels can be allocated to MTs that are far away from the BS, whereas the licensed channels can be allocated to MTs that are close to the BS; this channel-allocation policy will help maximize cell capacity. In addition, the opposite of this policy results in the max-min fair allocation. These findings are both analytically and experimentally verified.

The remainder of this paper is organized as follows. Section II discusses prior related work. Section III illustrates the legacy IEEE 802.22 system and presents our proposed model of CR-enabled cellular networks. In Section IV, we pro-

\footnotetext{
${ }^{2}$ Naturally, there could be multiple cellular service providers who want to share idle TV channels. In this case, if a cellular service provider already uses the channel, the other providers may regard it as if it were occupied by primary users. Otherwise, these providers may collaborate through a spectrum broker [9]. In our approach, we therefore consider a single cellular system only.

${ }^{3}$ Throughout this paper, we interchangeably use user and MT.

${ }^{4}$ To make only the partial area available for CR systems, particularly for the downlink, sector antenna or various directional antennas, such as beamforming, can be combined with our approach.
}

pose the collaborative sensing by exploiting already-deployed cellular BSs. Section V studies channel allocation in the CR-enabled cellular networks. Section VI presents the evaluation results for the proposed sensing and channel allocation. Finally, Section VII concludes this paper.

\section{RELATED WORK}

There has been extensive prior work on CR; for example, see the surveys in [8] and [9]. The most common CR architecture is to separately build a secondary network from a primary network as in IEEE 802.22 systems. If there is a spectrum broker [9] that gathers the available spectrum information, it may facilitate spectrum sharing among multiple CR networks. In case that there is no such information, secondary networks will opportunistically access the channel that is licensed to a primary network [10], [11].

The unique feature of our CR-enabled cellular networks is that both primary and secondary radio spectra are accessed by a single network. This feature has not yet been investigated elsewhere, although a concept of dual-mode spectrum sharing has been proposed, where a mobile user is equipped with CR capability to access a new CR network and existing networks [9], [12]. For 4G systems, the reconfigurability concept enables user equipment to dynamically select the most appropriate configurable action [13]. The reconfigurable radio networks that are conceptually described in [14] can be the ultimate goal of our network model.

Spectrum sensing is key to the implementation of CR systems, particularly when information on available radio spectrum is not provided. In [15]-[17], energy and feature detection techniques are introduced, and in [18], spectrum sensing for TV bands is studied. In addition, cooperative/collaborative-sensing issues have been studied; for example, see [19] and the references therein. In cooperative sensing, sensors can be chosen to minimize the number of sensors to use [20]. Although most of the state-of-the-art work assumes that sensors are arbitrarily distributed, we integrate the sensing functionality with alreadyinstalled cellular BSs.

Spectrum allocation in CR systems has also been covered in the literature (for example, see [21]-[23]). The authors in [21] investigated the question: "Which node should use how wide a spectrum band at what center-frequency and for how long?" They solved it by a centralized and a distributed protocol. In [22], channel assignment is considered to guarantee the interference constraints to PUs, and in [23], a distributed scheduling algorithm is presented. To the best of our knowledge, the problem of assigning both primary and secondary channels to the same set of users has not yet been addressed.

When cellular systems are based on orthogonal frequencydivision multiple access (OFDMA) as in Worldwide Interoperability for Microwave Access (WiMAX), they are exposed to intercell interference in cell-edge areas. Although this issue is not explicitly mentioned in this paper, any technique for intercell interference mitigation could be applicable to our system model. This issue is also discussed in the IEEE 802.22 standard [5] to support interference mitigation and coexistence, particularly when there are multiple BSs in the same area. 
Because our model is based on the existing cellular model, solutions that are developed for WiMAX and 3G LTE systems (e.g., [35] and [36]) can also be used for our model if both CR and licensed channels operate on the same OFDMA platform. For example, fractional frequency reuse [36], which dynamically assigns a greater frequency reuse factor to cell-edge users, is developed to enhance cell-edge performance. This frequency planning can be applied to CR channels without affecting our channel-allocation strategy.

\section{Network MOdel}

Here, we provide a brief overview of the existing network model of the IEEE 802.22 network and then describe the proposed network overlay model of CR-enabled cellular networks.

\section{A. IEEE 802.22 WRAN}

In IEEE 802.22 WRAN systems, a BS manages a number of consumer premise equipment (CPE) within its own cell as in cellular systems. Its air interface and Media Access Control (MAC) frame are very similar to the IEEE 802.16 system based on OFDMA and time-division duplexing (TDD). The spectral efficiencies are in the range of $0.5-5 \mathrm{~b} / \mathrm{s} / \mathrm{Hz}$ by using various modulation and coding schemes [5].

One important feature of this system is the sensing of a PU signal. In particular, the IEEE 802.22 standard defines distributed sensing, which enables various CPE to measure different TV channels and report the results to the BS [6]. The BS gathers all the sensed information and determines/estimates the availability of channels. Once the PU's presence on a channel has been detected by any CPE or the BS, the channel should quickly be vacated.

Because the coverage range of IEEE 802.22 WRANs is specified as $33 \mathrm{~km}$, a WRAN cell may cover dozens of BSs of an existing cellular network, of which the typical cell size is in the range of several hundred meters to several kilometers. The cell size has been reduced to a few hundred meters or several kilometers in 3G high-speed packet access (HSPA) and WiMAX systems, because the radio frequency shifts to above $2 \mathrm{GHz}$.

\section{B. CR-Enabled Cellular Network}

The proposed network model incorporates a CR system with an existing cellular system, and hence, no additional BS is needed for the CR system. Unlike the case where two networks (IEEE 802.16 and IEEE 802.22) separately operate, a cellular BS in the proposed model manages both spectrum bands that are explored by CRs and the original licensed bands. The role of spectrum sensing fulfilled by a WRAN BS and CPE in a distributed manner is handed over to the BSs of existing wireless systems. Additional sensors could also be attached to MTs, but this extension is not considered, assuming that, in the area of a WRAN BS, there will be a sufficient number of cellular BSs to perform spectrum sensing over TV bands. Additional sensors may be considered when radio spectra are accessible by PUs with a very narrow coverage, e.g., wireless microphones or Programmable Making and Special Events (PMSE) devices [24], [25]. In reality, the sensing of wireless microphones is a difficult issue [26]; therefore, one possible solution is to exclude the radio spectrum that is allocated to wireless microphones, and we do not treat this problem.

For spectrum sensing in the IEEE 802.22 standard, each transmission frame is divided into sensing and datatransmission periods [6], [19], [29]. All CPE and a WRAN BS do not transmit any data during the sensing period to perform spectrum sensing. Following this practice, in our network model, all BSs have a synchronized frame structure to perform spectrum sensing during the same sensing period.

Compared to the case where an IEEE 802.22 network separately operates, the proposed model exploits the following advantages of an existing cellular network: 1) The cellular service provider can provide additional bandwidth for end users and 2) in allocating channels to users, different characteristics of radio propagation over TV and cellular bands can be exploited. To effectively utilize these augmented channels of different characteristics, we will explore how we can allocate such heterogeneous channels in Section V.

Meanwhile, the proposed model exploits the following advantages in terms of operating a CR network. First, BSs that will perform sensing have been installed on a tall mast or the rooftop of a building; therefore, the sensing performance will be much higher than the CPE-based sensing of the IEEE 802.22. Second, because BSs are interconnected through a wired backhaul network, the information that is sensed by each BS can easily be disseminated over the backhaul network, which alleviates the problem of legacy cooperative/collaborative sensing that radio resources should be consumed for the exchange of sensed information [29]. Third, the network can also use some licensed frequency bands for its original intended operation; therefore, there is no need for establishing a separate common control channel, which has been a controversial issue in configuring CR systems [27]. Fourth, because the cell size of a CR network that was set to $33 \mathrm{~km}$ in WRANs is decreased to as small as the cell size of an existing cellular system, the transmit power can significantly be reduced; thus reducing the interference to PUs. ${ }^{5}$ This issue will further be discussed in Section V. Finally, the area where a CR channel is accessible is expanded. In IEEE 802.22 WRANs, the whole cell area should be forbidden from accessing a channel if a PU is detected on that channel anywhere in the cell. However, when the cell size is small, BSs in some areas may still allow their users to access the channel, whereas the other BSs do not, as shown in Fig. 1. Based on these ideas, we develop a new collaborative sensing mechanism, which will be described in the next section.

\section{Collaborative Sensing Based on Cell Topology}

We now describe the proposed collaborative sensing scheme based on cell topology.

\footnotetext{
${ }^{5}$ Although the LTE systems may support a longer cell size up to $100 \mathrm{~km}$, we consider a general system of which cell sizes are several kilometers.
} 


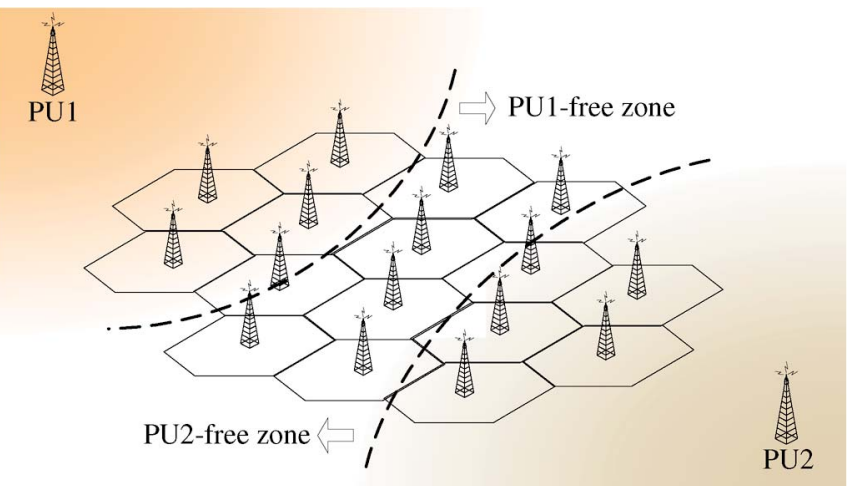

Fig. 1. Increased resource granularity in the proposed network model.

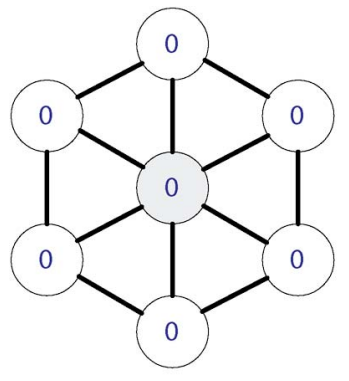

Complete PU-free

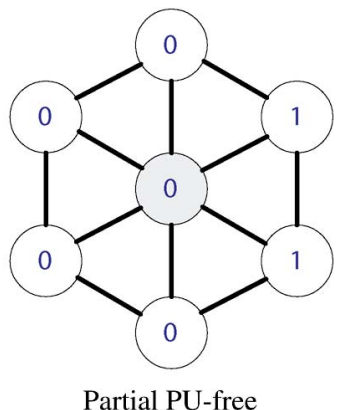

Partial PU-free
Fig. 2. Examples of collaborative sensing based on cell topology.

\section{A. Procedure}

1) Obtain the Topology of BS Sensors: First, each BS needs to obtain the topology of its neighboring BSs to collaborate with. This information is easily obtained in conventional cellular networks, i.e., by exchanging the location information of each BS through the backhaul network or by reports from MTs on the neighboring BSs. The latter approach is made available by a handoff procedure, because a server BS can learn from MTs the information on the neighboring BS to which an MT moves. Thus, each BS can obtain the topology information of neighboring BSs; for example, when there are six neighboring BSs as in the conventional hexagonal cellular model, they are linked with a centered BS, as shown in Fig. 2.

2) Categorize MTs Into Zones of Effective BSs: In most cellular networks, an MT keeps track of pilots that are broadcast by neighboring BSs for several purposes. The most common purpose is to support handoffs: each MT must know the identification (ID) number of a neighboring BS that broadcasts the most dominant signal strength, which is a candidate cell to move to. In addition, the neighboring BSs need to be known due to the advent of recent techniques, e.g., fractional frequency reuse, macrodiversity, and beamforming, which require the cooperation of two or more BSs [28]. To exploit these techniques, an MT needs to report the channel information from the dominant neighboring BS(s) and its ID. Dominant BSs that are shown by an MT are called effective BSs. Thus, a BS can categorize MTs into zones of effective BSs, as shown in Table I, where $X_{i}$ means the sensing result of BS $i$. As shown in the example, when $X_{1}=X_{4}=0$ (i.e., no PU is detected) and $X_{2}=X_{3}=1$ (the PU is detected), MTs who report them
TABLE I

EXAMPLE OF CATEGORIZING MTS

\begin{tabular}{|c|c|c|c|}
\hline Effective & \multirow{2}{*}{ MTs } & \multicolumn{2}{|c|}{ Partial PU-free decision $Y_{1}$} \\
\cline { 3 - 4 } BSs & & $X_{2}=1$ & $X_{2}=X_{3}=1$ \\
& & $X_{1}=X_{3}=X_{4}=0$ & $X_{1}=X_{4}=0$ \\
\hline 1 (serving BS) & & 0 & 0 \\
1,2 & 1 & 1 \\
1,3 & & 0 & 1 \\
1,4 & $\ldots$ & 0 & 0 \\
$1,2,3$ & & 1 & 1 \\
$1,2,4$ & & 1 & 1 \\
$1,3,4$ & & 0 & 1 \\
\hline
\end{tabular}

as effective BSs can access the channel. MTs that listed "1, 4" as their effective BSs are probably located in a cell-edge area between BSs 1 and 2. However, MTs that listed "1, 2" as their effective BSs are prohibited from accessing the channel, because they are possibly located to BS 2, which declared PU occupied. This categorization table will be maintained by using reports from MTs.

3) Exchange the Sensed Information Between Neighboring BSs Through Backhaul Networks: Each BS performs spectrum sensing and exchanges the sensed information with its neighboring BSs in the topology. A decision on whether a PU currently utilizes a specific channel can be made by one of the well-known detection schemes, e.g., matched filter coherent detection, energy detection, and cyclostationary feature detection [15], [16] or a combination thereof [17]. All of the BSs should simultaneously perform sensing on a specific channel during the sensing period and share a list of such channels, including channels that are currently in use and other potential channels that may be used in the future.

4) Declare PU-Free Zones: Based on the sensed information from its neighboring BSs, each BS can declare its cell area to be completely PU-free, partially PU-free, or PU occupied for each channel. In case of PU occupied, the BS does not use the channel, and in case of completely PU-free, the BS assigns the channel to be accessed in its area. In case of partially PU-free, the BS allows only some MTs to access the channel. The decision mechanism will be described next.

\section{B. Decision Mechanism}

We propose the following two decision mechanisms: one approach for completely PU-free and another approach for partially PU-free, where the final decision is made according to the sensing results from a BS and the neighboring BSs. By using " 0 " and "1" to represent the absence and presence of a PU, respectively, we can express the final decision $Y_{i}$ of BS $i$ as a function of the sensing result of BS $i, X_{i}$, and the sensing results of neighboring BSs, $X_{j}$ 's as

$$
Y_{i}=X_{i} \times \prod_{j \in \mathbb{N}_{i}} X_{j}
$$

where $\mathbb{N}_{i}$ represents the set of neighboring BSs of BS $i$. That is, $Y_{i}=0$ means that this channel is accessible, whereas $Y_{i}=1$ means that it is inaccessible due to the presence of a PU. 
TABLE II

Decision by the Proposed Collaborative Sensing

\begin{tabular}{|c|c|c|}
\hline Self-result & Result of neighboring BSs & Decision \\
\hline$X_{i}=0$ & $\sum_{j \in \mathbb{N}_{i}} X_{j}=0$ & Complete free \\
$X_{i}=0$ & $\sum_{j \in \mathbb{N}_{i}} X_{j} \leq \delta_{i}$ & Partial free \\
$X_{i}=1$ & $\sum_{j \in \mathbb{N}_{i}} X_{j} \geq\left|\mathbb{N}_{i}\right|-\varepsilon_{i}$ & Occupied \\
\hline$X_{i}=1$ & $\sum_{j \in \mathbb{N}_{i}} X_{j} \leq \delta_{i}$ & False alarm \\
$X_{i}=0$ & $\sum_{j \in \mathbb{N}_{i}} X_{j} \geq\left|\mathbb{N}_{i}\right|-\varepsilon_{i}$ & Misdetection \\
\hline
\end{tabular}

1) Completely PU-Free: A cell area is declared to be completely PU-free when neither the BS nor neighboring BSs detect any PU. One example of a PU-free zone is shown in Fig. 2, where the BS at the center is declared to be PU-free, because its six neighboring BSs and itself do not detect any PU. In such a case, communication within the centered cell is unlikely to interfere with any PU, because its neighboring BSs do not detect any PU either.

2) Partially PU-Free: It may become inefficient to keep the entire cell area from accessing a channel when only some of the neighboring BSs report the presence of a PU on that channel. For example, Fig. 2 shows that only two neighboring BSs report 1 for a channel, whereas the other BSs find the channel available. That channel may be allocated to some MTs, unless they are close to those BSs. ${ }^{6}$ The information on such MTs is given by the table of MTs categorized by effective BSs, as shown in Table I. This case is declared to be partially PU-free. In case of partially PU-free, $\mathbb{N}_{i}$ in (1) means the set of effective BSs.

3) Decision Using Regional Correlation: In general, spectrum sensing is imperfect due to the intrinsic nature of sensing that relies on signal strength. PU-free may be declared even in the presence of a PU, and this case is called a misdetection. On the other hand, PU occupied may be declared in the absence of PUs, triggering a false alarm. To enhance the sensing performance by preventing misdetections and false alarms, various sensing techniques have been proposed, and one key technique is found in collaborative sensing. For example, the AND rule is to declare the presence of a PU if all the sensors detect (or do not detect) a PU, and the OR rule is to do the same if at least one sensor detects (or does not detect) a PU (see [19] and references therein for more details on this case).

Each BS may predict whether its detection is correct by observing the results from its neighboring BSs, because there will be a regional correlation among the BSs. Therefore, we propose the use of regional correlation to enhance decision correctness. For example, if a certain BS detects a PU, whereas most of its neighboring BSs do not, the decision may be suspected to be a false alarm. The number of such neighboring BSs is $\left|\mathbb{N}_{i}\right|-\delta_{i}$, where $\delta_{i}$ represents the number of unsuspected neighboring BSs. Similarly, if a certain BS does not detect any PU whereas most of the neighboring BSs do, the decision may be suspected of misdetection. The number of such neighboring BSs is $\left|\mathbb{N}_{i}\right|-\varepsilon_{i}$, where $\varepsilon_{i}$ represents the number of unsuspected neighboring BSs. In both cases, the BS may reverse its decision. These scenarios are summarized in Table II.

\footnotetext{
${ }^{6} \mathrm{Here}$, we consider omnidirectional antennas, but the BS may use sector antennas (or beamforming) to further reduce possible interference to a PU.
}

For reasonable formation of regional correlation, the range of $\delta_{i}$ and $\varepsilon_{i}$ is derived as follows:

$$
0 \leq \delta_{i}, \varepsilon_{i} \leq\left\lceil\frac{\left|\mathbb{N}_{i}\right|-1}{2}\right\rceil-1
$$

When $\delta_{i}$ or $\varepsilon_{i}$ is 0 , it implies that all the neighboring BSs report the same sensing result. This case is equivalent to the AND/OR rule. On the other hand, when $\delta_{i}$ or $\varepsilon_{i}$ is $\left\lceil\left(\left|\mathbb{N}_{i}\right|-\right.\right.$ $1 / 2)\rceil-1$, it implies that the majority of BSs, including itself, report the same sensing result. This case is equivalent to the majority rule. For example, when $\mathbb{N}_{i}=6$, the maximum of $\delta_{i}$ or $\varepsilon_{i}$ is 2 , because three BSs, including the BS at the center, report different results from the other four neighboring BSs. When $\mathbb{N}_{i}=7$, the total number of BSs is 8 , including the BS at the center; therefore, the majority is 5 , which means that $\delta_{i}$ or $\varepsilon_{i}$ should be 2 , excluding the centered BS.

In summary, when regional correlation is used, we can express the final decision as follows:

1: $\quad$ PU occupied is declared, if $\sum_{j \in \mathbb{N}_{i}} X_{j}-\left|\mathbb{N}_{i}\right|+\varepsilon_{i} \geq 0$

2: Completely PU-free is declared, if $\sum_{j \in \mathbb{N}_{i}} X_{j}=0$

3: Otherwise, partially PU-free is declared.

In this paper, we simply assume that a decision of each neighboring BS has the same quality. Their locations can differently be quantified for the final decision, which would be an interesting issue to explore.

\section{Channel Allocation}

\section{A. Problem Formulation}

In our network model, we have the following two sets of channels: 1) the original set of channels $\mathbb{F}$ that are licensed to this system and 2) the set of channels $\mathbb{G}$ that are available through our CR enabler. Then, the question is how we can allocate these two sets of channels to mobile end users in each cell.

To answer this question, we need to understand the intrinsic nature of carrier frequency. A radio spectrum is known to propagate with a diffraction effect, and the propagation distance becomes shorter as the carrier frequency becomes higher in the same environment. Therefore, the path loss of TV bands around several hundred megahertz is lower than cellular bands around $2 \mathrm{GHz}(3 \mathrm{G})$ or 2.3 or $3.5 \mathrm{GHz}$ (WiMAX). The characteristics of various spectrum bands have been verified through field tests; for example, see the Okumura-Hata model [30]. Cellular systems are also known to suffer more path loss, and the pathloss exponent lies between 3 and 5, whereas it is found to lie between 2.0 and 3.3 in very high frequency (VHF)/UHF bands [31], [32].

In allocating channels of different carrier frequencies to users (i.e., MTs), we first want to maximize the cell capacity, which is equivalent to the throughput maximization, when the bandwidth 
is evenly distributed to users. The strategy of maximizing the cell capacity is expressed as follows:

$$
\begin{array}{r}
\text { Objective I : } \quad \max \sum_{k} C_{k} \\
\text { subject to } \lambda_{1}=\lambda_{2}=\cdots
\end{array}
$$

where $C_{k}$ is the average capacity per unit bandwidth of user $k$, and $\lambda_{k}$ is the fraction of the total bandwidth that is allocated to user $k$.

In addition, we can consider other objectives such as max-min allocation as follows:

$$
\begin{array}{r}
\text { Objective II : } \quad \max \min _{k} C_{k} \\
\text { subject to } \lambda_{1}=\lambda_{2}=\cdots .
\end{array}
$$

For our analysis, we define several symbols as follows.

- $L$ : the number of licensed channels;

- $V$ : the number of TV channels that are declared to be PU-free in the cell;

- $f_{i}$ : a licensed channel frequency $(i=1,2, \ldots, L)$;

- $g_{j}$ : a TV channel frequency $(j=1,2, \ldots, V)$;

- $\lambda_{k}\left(f_{i}\right)$ : fraction of bandwidth that is used by a licensed frequency $f_{i}$;

- $\lambda_{k}\left(g_{j}\right)$ : fraction of bandwidth that is used by a TV frequency $g_{j}$.

Note that $\lambda_{k}=\sum_{i} \lambda_{k}\left(f_{i}\right)+\sum_{j} \lambda_{k}\left(g_{j}\right)$. Furthermore, we define

$$
\beta_{k}=\sum_{i=1,2, \ldots, L} \lambda_{k}\left(f_{i}\right) .
$$

The sum of all the fractions of bandwidth should be 1 , i.e., $\sum_{i=1,2, \ldots, L} \lambda_{k}\left(f_{i}\right)+\sum_{j=1,2, \ldots, V} \lambda_{k}\left(g_{j}\right)=1$; therefore, we obtain

$$
1-\beta_{k}=\sum_{j=1,2, \ldots, V} \lambda_{k}\left(g_{j}\right) .
$$

Then, $C_{k}$ is written as

$$
C_{k}=\sum_{i=1,2, \ldots, L} \lambda_{k}\left(f_{i}\right) C_{k}\left(f_{i}\right)+\sum_{j=1,2, \ldots, V} \lambda_{k}\left(g_{j}\right) C_{k}\left(g_{j}\right)
$$

where $C_{k}\left(f_{i}\right)$ and $C_{k}\left(g_{j}\right)$ represent user $k$ 's capacities, each achievable at frequencies $f_{i}$ and $g_{j}$. Here, user $k$ 's capacity at channel $x$ is expressed by the Shannon capacity as

$$
C_{k}(x)=\log \left(1+\operatorname{SNR}_{k}(x)\right) .
$$

In particular, the signal-to-noise ratio (SNR) that is measured at $g_{j}$ may include interference $I_{p}$ from a PU. For the operation of a CR network, FCC imposes an SNR requirement when the signal is measured from a PU. For example, no PU is assumed present if the SNR is less than $-116 \mathrm{dBm}$ for digital television (DTV) signal detection [6]. This threshold is even less than the typical noise level $N=-96 \mathrm{dBm}$; therefore, we can assume $I_{p}+N \approx N$ on $g_{j}$ in PU-free areas, and henceforth, $I_{p}$ will not be considered.
The noise may include adjacent channel interference, particularly for TV channels, when an adjacent channel is used. This problem is solved by vacating both adjacent channels [6]; therefore, we do not consider adjacent channel interference.

Now, the noise in an SNR consists of intercell interference and background noise. We consider the following two cases.

- Case 1. For PU-free cells for a certain TV channel $g_{j}$, the sum of the intercell interference is greater than the background noise and is dominant in determining an SNR.

- Case 2. The background noise is greater than the sum of the intercell interference and is dominant in determining an SNR.

To derive a solution for channel allocation, we make the following assumptions. Because the frequency band of any licensed channel is always greater than the frequency band of any TV channel, it is written as follows.

Assumption 1: $f_{i}>g_{j}, \forall i=1,2, \ldots, L$ and $j=1,2, \ldots, V$.

Assumption 2: In our network model, each cell utilizes all available channels, i.e., every cell that declares $g_{j}$ to be PU-free uses $g_{j}$ and $f_{1}, f_{2}, \ldots, f_{L}$. In particular, when $g_{j}$ is completely PU-free, the set of neighboring cells $\mathbb{N}_{i}$ of this channel is the same as in the other frequency $f_{i}$ 's. To support these channels, a BS in our system should be equipped with two transmitters, each for $f_{i}$ and $g_{j}$.

For theoretical tractability, we do not consider power control for each channel.

Assumption 3: The transmit power over each of $f_{1}, f_{2}$, $\ldots, f_{L}$ or $g_{j}$ is fixed for all cells. However, the transmit power at $f_{i}$ may be different from (will be greater than) the transmit power at $g_{j}$.

\section{B. Channel-Allocation Solution}

In case 1 , where intercell interference is dominant compared to background noise, users achieve lower capacity when accessing a TV channel, as stated in the following theorem.

Theorem 1: In case 1, under Assumptions 1-3, each user achieves higher capacity if he/she only accesses a licensed channel and not a TV channel that is completely PU-free. In the case of (5), $\beta_{k}=1$.

The proof is given in Appendix A.

On the contrary, in case 2, where the background noise is greater than the intercell interference or there is a single cell, users will achieve higher capacity if they access only a TV channel and the transmit power over all channels is the same. This case is easily predictable, because TV channels are less attenuated due to the nature of low carrier frequency. This condition is stated in the following corollary.

Corollary 1: In case 2, under Assumptions 1-3, with the same transmit power, each user achieves higher capacity if it only accesses a TV channel and not a licensed channel. In the case of (5), $\beta_{k}=0$.

In case 1 , all the users will want to access $f_{1}, f_{2}, \ldots, f_{L}$ to achieve high capacity, and in case 2 (same transmit power), all the users will want to access $g_{1}, g_{2}, \ldots, g_{V}$ to achieve high capacity. For fair distribution of bandwidth of $f_{i}$ 's and $g_{j}$ 's, 
some users may have to be assigned $\beta_{k}<1$ in case 1 and $\beta_{k}>0$ in case 2 .

Our solution to the maximization problem Objective I is given as follows.

Theorem 2: Under Assumptions 1-3, when users in a cell are sorted such that $d_{1}>d_{2}>\cdots>d_{K}$, where $d_{k}$ is the distance between user $k$ and the BS, the maximization of cell capacity is achieved by dividing users into two groups. In one group, $\beta_{k}=0$ for $k=1, \ldots, \kappa-1$, and in the other group, $\beta_{k}=1$ for $k=\kappa+1, \ldots, K$ ( $\kappa$ may belong to either group or both).

The proof is given in Appendix B. This theorem states that low-carrier-frequency channels such as TV bands should be allocated to users who are far away from the BS. In reality, cells have different sizes and shapes, and the distance from the BS is estimated by the SNR rather than the geographical distance. Therefore, $d_{k}$ should be interpreted as the effective distance measured by their SNR.

Based on Theorems 1 and 2, we can easily derive the solution to Objective II, which is exactly reversed to Objective I. For example, if there are two users with $d_{1}>d_{2}$ and two channels $f_{i}$ and $g_{j}$, users 1 and 2 should be assigned $f_{i}$ and $g_{j}$, respectively, to meet Objective II. This proof is similarly given as in the aforementioned theorem; therefore, we omit it.

Corollary 2: Under Assumptions 1-3, when users in a cell are sorted such that $d_{1}>d_{2}>\cdots>d_{K}$, where $d_{k}$ is the distance between user $k$ and the BS, Objective II is achieved by dividing users into two groups. In one group, $\beta_{k}=1$ for $k=1, \ldots, \kappa-1$, and in the other group, $\beta_{k}=0$ for $k=\kappa+$ $1, \ldots, K$ ( $\kappa$ may belong to either group or both).

Note that Theorem 1 may not hold for partially PU-free cells, because some neighboring cells do not use $g_{j}$, and thus, certain users may achieve higher capacity at $g_{j}$ than at a licensed channel. However, the number of such users is small, because most of the users who are allowed to transmit at $g_{j}$ in a partially PU-free cell are not located near a neighboring BS that is declared to be PU occupied. Therefore, Theorem 2 can be effective for partially PU-free cells, and this case is verified through simulation in Section VI. In partial-PU free areas, an empty TV channel should carefully be accessed to avoid interfering with any PUs. This issue will be discussed next.

\section{Boundary Condition}

In IEEE 802.22-WRAN systems, the transmit power of BSs may go up to $100 \mathrm{~W}$, but CPE uses $4 \mathrm{~W}$; therefore, the transmit power of BSs will be set between 4 and $100 \mathrm{~W}$ [33]. In our system model, each BS and MTs may consume less transmit power, because the coverage of cellular systems is much smaller than WRAN systems. Let $R_{\text {wran }}$ and $R_{\text {cell }}$ be the radius of a BS in WRAN and cellular systems, respectively. We can set transmit power $p_{s}$ over $g_{j}$ such that a cell-edge user at distance $R_{\text {cell }}$ in our system senses the receive power not greater than a cell-edge user at distance $R_{\text {wran }}$ in the WRAN system as

$$
p_{s} R_{\text {cell }}^{-\alpha_{s}} c \leq p_{\text {wran }} R_{\text {wran }}^{-\alpha_{s} c}
$$

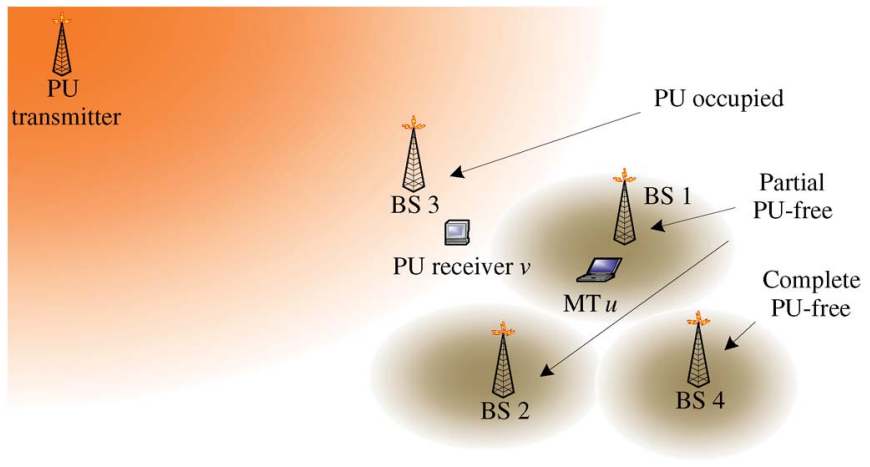

Fig. 3. Scenario of interference to a potential PU in boundary cells.

TABLE III

PARAMETERS FOR EVALUATION

\begin{tabular}{|c|c|}
\hline Path loss exp. of TV bands $\left(\alpha_{s}\right)$ & 2.5 \\
Path loss exp. of cellular bands $\left(\alpha_{o}\right)$ & 3.5 \\
Shadowing deviation & $6 \mathrm{~dB}$ \\
Radius of a cell $\left(R_{\text {cell }}\right)$ & $3 \mathrm{Km}$ \\
Transmit power of BSs at TV bands $\left(p_{s}\right)$ & $125 \mathrm{~mW}$ \\
Power of a TV transmitter & $40 \mathrm{~W}$ \\
PU-detection threshold & $-116 \mathrm{dBm}$ \\
Number of simulations & 1000 \\
\hline
\end{tabular}

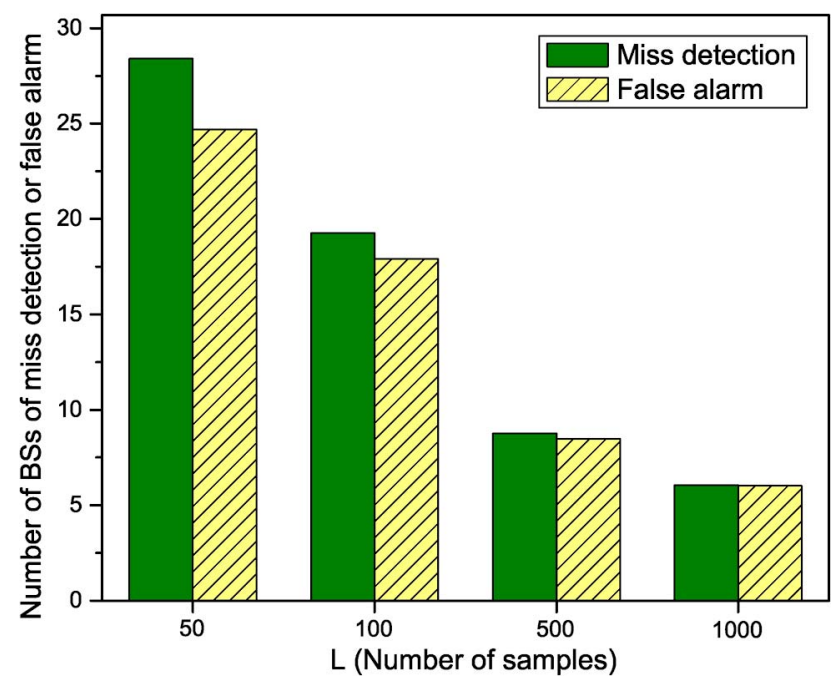

Fig. 4. Number of BSs with misdetections or false alarms as a function of $L$.

where $p_{\text {wran }}$ represents the transmit power of WRAN systems, $c$, a path loss constant, and $\alpha_{s}$, a path-loss exponent over $g_{j}$. For example, when $\alpha_{s}=2.5, R_{\text {wran }}=33 \mathrm{~km}, R_{\text {cell }}=3 \mathrm{~km}$, and $p_{\text {wran }}=40 \mathrm{~W}$, assuming that the antenna efficiency is the same for both cases, $p_{s}$ is at most $100 \mathrm{~mW}$. This value is even much less than $4 \mathrm{~W}$, which is the transmit power of CPE in WRANs.

Because the value of $p_{s}$ will affect the performance of a PU, it should be determined by considering how much a PU (possibly a PU receiver) beyond any PU-free area may be interfered with by PU-free cells. One example of such a scenario is shown in Fig. 3, where BSs 1 and 2 are partially PU-free, and there is a PU receiver $v$ in the PU-occupied cell of BS 3, which is a neighbor to BSs 1 and 2. We found that less interference 
TABLE IV

Probability of Misdetections or False Alarms When Regional Correlation Is Used

\begin{tabular}{|c||c|c|c||c|c|c|}
\hline \multicolumn{1}{|c||}{$\delta$ or $\varepsilon$} & \multicolumn{3}{c||}{ Misdetection } & \multicolumn{3}{c|}{ False alarm } \\
\cline { 2 - 6 } & total & $\begin{array}{c}\text { reversed } \\
\text { decision }\end{array}$ & $\begin{array}{c}\text { wrong } \\
\text { decision }\end{array}$ & total & $\begin{array}{c}\text { reversed } \\
\text { decision }\end{array}$ & $\begin{array}{c}\text { wrong } \\
\text { decision }\end{array}$ \\
\hline 0 & 1.58 & 0.17 & 0.005 & 1.46 & 0.14 & 0.002 \\
1 & 1.60 & 0.61 & 0.03 & 1.46 & 0.34 & 0.007 \\
2 & 1.57 & 1.36 & 0.29 & 1.47 & 0.51 & 0.02 \\
\hline
\end{tabular}

is generated by PU-free BSs in our network model than by a WRAN BS if the following condition is met:

$$
p_{s} \leq \frac{1}{n} p_{\text {wran }}\left(\frac{R_{\text {wran }}}{R_{\text {cell }}}\right)^{-\alpha_{s}}
$$

where $n$ is the number of neighboring partially PU-free BSs seen by a PU ( $n$ is 2 in the case in Fig. 3), and hence, the aggregated receive power in the worst case is $n p_{s} R_{\text {cell }}^{-\alpha_{s}}$. BSs in a partially PU-free area, e.g., BSs 1 and 2 in Fig. 3, are probably the main interferer to a PU $v$, because a certain MT $u$, even if it is closer to the PU (but is located in a cell-interior area), will use much less transmit power for uplink than downlink transmissions. Accordingly, we prove the downlink case in the following theorem.

Theorem 3: When the condition of transmit power satisfies (10) with Assumptions 1-3, PU-free BSs in our model cause less interference to PUs beyond a PU-free area than a WRAN BS.

The proof is given in Appendix C.

\section{Numerical Evaluation}

We conducted simulation experiments to evaluate the collaborative sensing based on cell topology and the proposed channel-allocation strategy. For a channel model, path-loss exponents are set to 2.5 and 3.5 for TV and cellular bands, respectively, and hexagonal BSs with a radius of $3 \mathrm{~km}$ are placed in an area of $200 \mathrm{~km} \times 200 \mathrm{~km}$. A TV transmitter is located at a point of the quadrangle area. The boundary of TV transmission is observed between 150 and $200 \mathrm{~km}$ from the TV transmitter. TV receivers will be located within this boundary, where this channel is not used for the cellular system. We do not show results in the case of other path-loss exponents or various cell radii, because the results shown here represent general performance. We also considered lognormal shadowing with 0 mean and $6 \mathrm{~dB}$ of deviation. We only use $40 \mathrm{~W}$ for a TV transmit power, because the other case of $20 \mathrm{~W}$ causes less interference to our system. The parameters that are used for our evaluation are listed in Table III.

\section{A. Evaluation of Collaborative Sensing Based on Cell Topology}

When there is no shadowing effect, the number of completely and partially PU-free BSs is 303 and 57, respectively, of a total of 1235 . When the shadowing (slow-fading) effect makes the channel condition fluctuate, it is difficult for boundary BSs to determine whether there is a PU. The fading effect is removed, although not completely, by averaging several (e.g., $L$ ) samples. ${ }^{7}$ As $L$ increases, detecting a PU becomes reliable, i.e., the number of false alarms or misdetections decreases. This result is confirmed in Fig. 4. When the frame length is $20 \mathrm{~ms}$ and $L$ is 100 , we need sampling for $2 \mathrm{~s}$, which is the same as the channel detection time, according to the FCC's regulatory guideline for the 5-GHz band [34]. Hence, in practice, it is hard to make $L$ longer, and we thus set $L=100$ as the default.

The result of the regional correlation is presented in Table IV. Because the number of neighboring BSs is six, i.e., $|\mathbb{N}|=6$, the range of $\delta$ and $\varepsilon$ is between 0 and 2 according to (2). We also conducted simulation experiments for $\delta=\varepsilon=3$, but the experiments did not yield any reasonable performance, because too many BSs unnecessarily reverse their decisions. When $\delta=0$, the probability of producing a misdetection is less than $2 \%$, and the probability that BSs reverse their decisions due to regional correlation is $0.17 \%$. When $\delta=2$, i.e., the majority rule is used, the probability that BSs reverse their decision is $1.36 \%$, and the probability that the reversed decision is wrong is $0.29 \%$. As shown in Table IV, the regional correlation is more effective for reversing misdetections than false alarms, and the majority rule $(\delta=\varepsilon=2)$ outperforms the AND/OR rule $(\delta=\varepsilon=0)$.

Furthermore, to reduce the events of misdetections and false alarms, our solution based on regional correlation can be combined with other techniques. Simply, the detection threshold (typically $-116 \mathrm{dBm}$ ) can be lowered, but this approach is effective only for lowering misdetection.

\section{B. Evaluation of Channel Allocation}

We now present the performance of channel allocation for a single completely PU-free cell when intercell interference is generated by two-tier neighboring cells. This scenario is found to be case 1, because the intercell interference is greater than the background noise. Transmit powers at TV bands and cellular bands are set to $125 \mathrm{~mW}$ and $20 \mathrm{~W}$, respectively. The cumulative density function (cdf) of spectral efficiencies at both channels is depicted in Fig. 5. Overall, the cellular channel achieves a larger spectral efficiency than the CR channel for case 1 , which confirms Theorem 1 . To confirm Corollary 1 for case 2, we also obtained the cdf of spectral efficiencies in both channels when there is no intercell interference and the transmit power is set to $5 \mathrm{~W}$. Overall, spectral efficiencies are higher, compared with case 1 . As expected, the CR channel achieves a larger spectral efficiency than the cellular channel.

\footnotetext{
${ }^{7}$ Although fast fading is unlikely to appear between a BS and a TV transmitter, it can be averaged out in a single sensing period when the duration of the sensing period is sufficient [29]. However, slow fading should be averaged over multiple sensing periods. Therefore, slow fading is the primary concern here.
} 


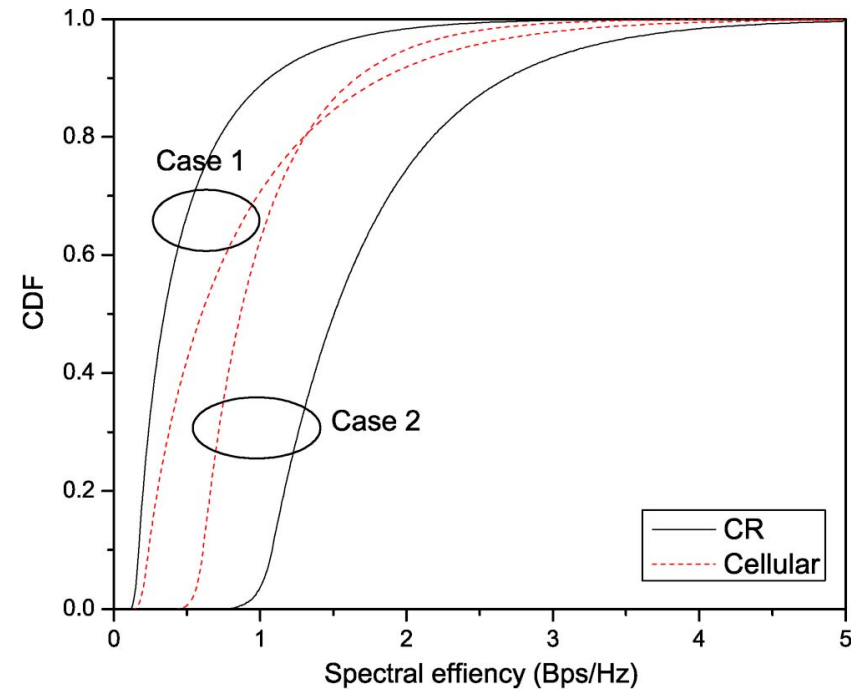

Fig. 5. CDF of spectral efficiency at CR and cellular channels (case 1).

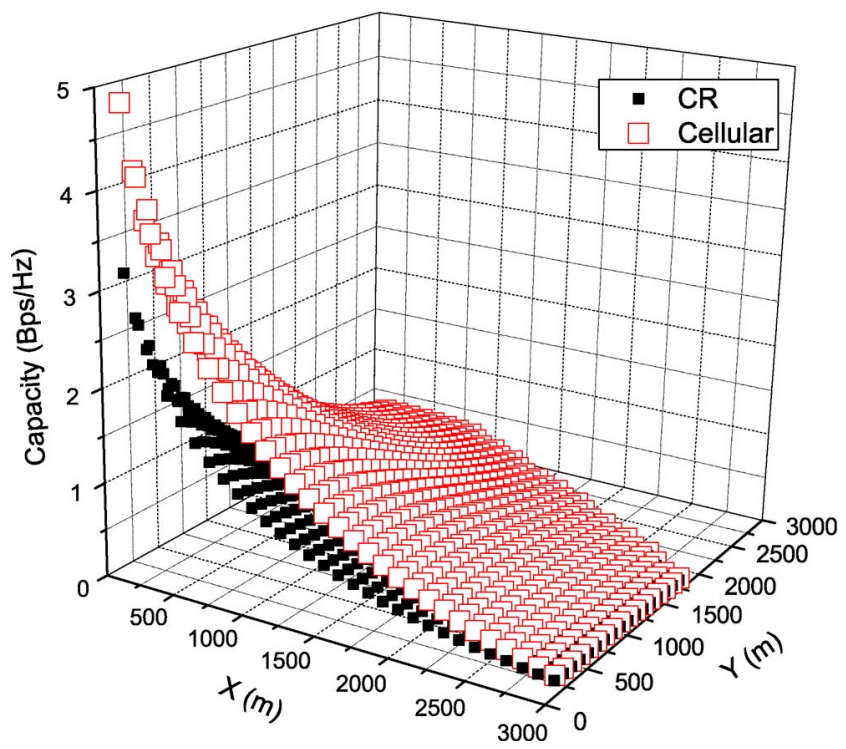

Fig. 6. Capacity at CR and cellular channels.

Theorem 1 is also verified in Fig. 6, which depicts the capacity achieved at each location for the two channels. The capacity of a cellular channel is greater than a CR channel everywhere in the cell. In addition, the capacity of a cellular channel more steeply decreases than a CR channel as the distance from the BS increases. This case confirms Theorem 2, because for any two locations 1 and 2, $C_{2, o}-C_{2, s}$ is greater than $C_{1, o}-C_{1, s}$, as proven in Appendix B.

The capacity sum is shown in Fig. 7 for cases 1 and 2, respectively. In this experiment, 20 users are randomly generated in a cell, and a half of these users are assigned a CR channel, whereas the other users are assigned a cellular channel over a downlink. The total capacity is averaged over 10000 experiments. Objective I achieves the highest capacity sum compared with the other two schemes. In the Random scheme, half of the users are randomly selected to be assigned a CR channel. In the Objective II scheme, channel allocation is exactly opposite to the proposed allocation, i.e., users near the BS are assigned a

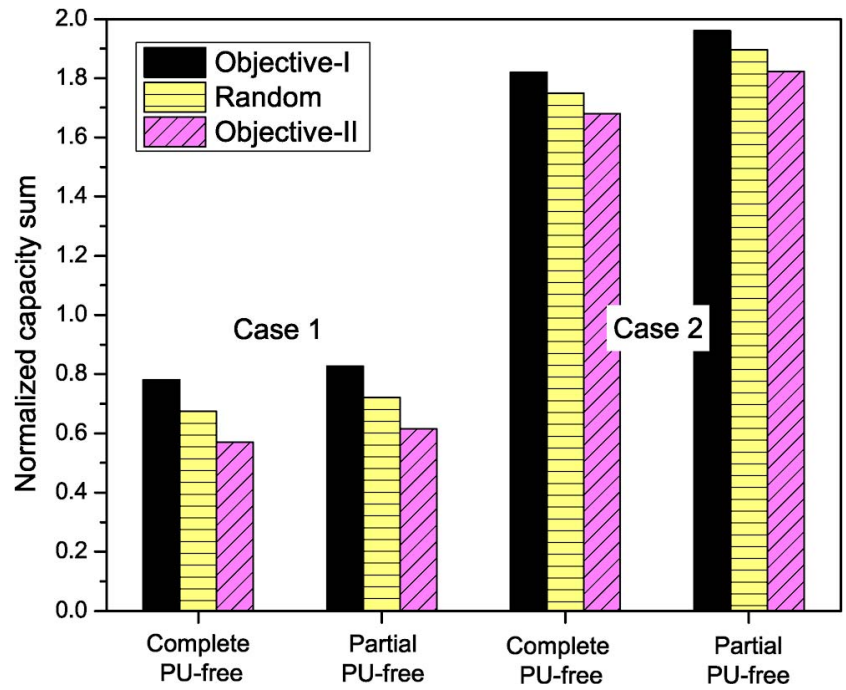

Fig. 7. Capacity sum in a completely and a partially PU-free cell for three channel-allocation schemes (case 1).

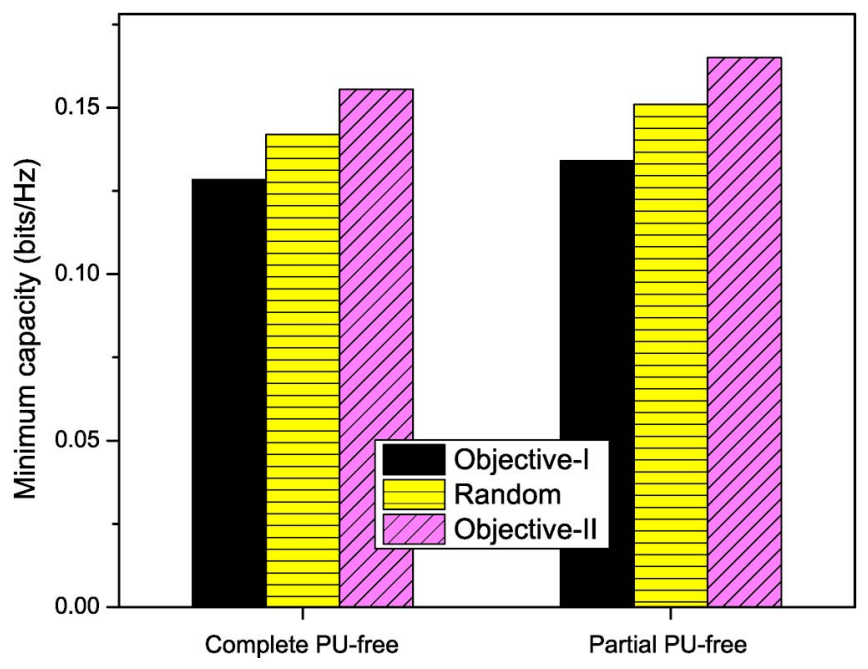

Fig. 8. Minimum capacity for three channel-allocation schemes (case 1).

CR channel, and users far from the BS are assigned a cellular channel. Fig. 8 shows that the minimum capacity is maximized in the Objective II scheme. We conducted the same experiment for a partially PU-free cell, where only four neighboring BSs are PU-free. Our results show that the two channel-allocation objectives work well for this partially PU-free cell. The overall capacity sum is slightly larger than in a completely PU-free cell, because the number of neighboring cells as interferers is reduced in the partially PU-free case.

Finally, in Fig. 9, we plot the amount of interference to a PU beyond a partially PU-free cell due to the channel usage in PU-free areas. The amount of interference is compared to the interference generated by a WRAN BS. According to (10), $p_{s}$ is $125 \mathrm{~mW}$ when $p_{\text {wran }}$ is $100 \mathrm{~W}$. Fig. 9 confirms Theorem 3, because the amount of aggregated interference that is generated by the partially PU-free BS at $(0,0)$ and the other BSs at $(x, y)$ for $x<0$ and $y<0$ is less than the interference that is generated by a WRAN BS that is located $30 \mathrm{~km}$ away from the partially PU-free BS. It is also smaller than the interference 


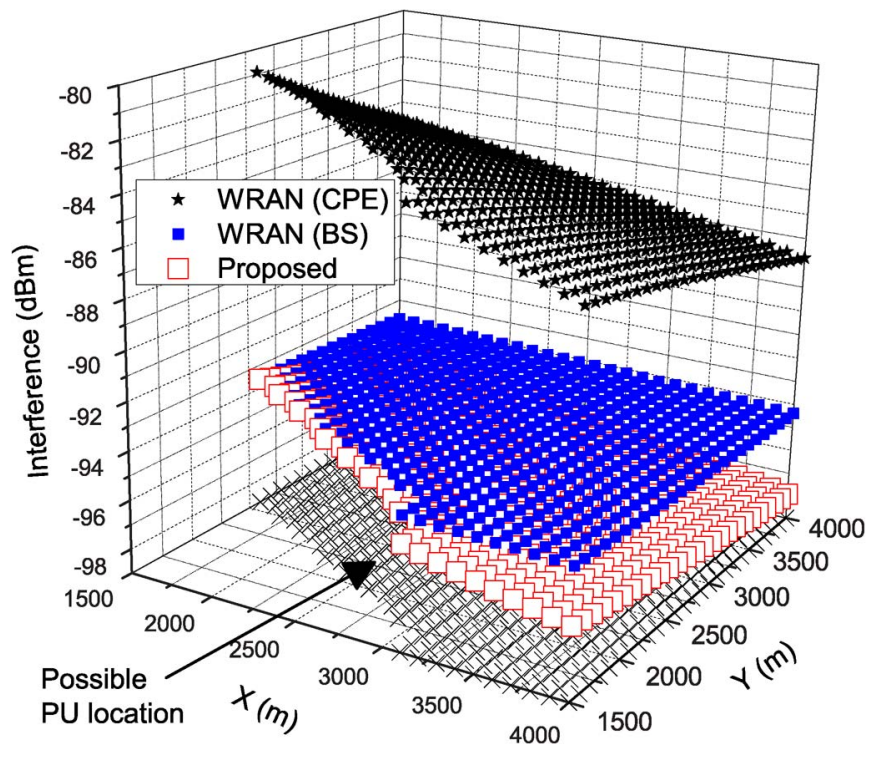

Fig. 9. Interference to a PU in the area beyond a partially PU-free cell.

that is generated by a WRAN CPE that is located at $(0,0)$ with a transmit power of $4 \mathrm{~W}$.

\section{CONCLUSION}

To increase the throughput of cellular systems that evolve toward $4 \mathrm{G}$, we have proposed a new way of integrating a CR system with an existing cellular network, thus overcoming the scarcity of radio spectrum. We have also proposed to augment the function of BSs to perform collaborative sensing. Using the proposed collaborative sensing mechanism, cells can be categorized as PU-free or PU occupied for each channel. The cellular system can therefore access an unused TV band and the original licensed band. An unused TV band should be allocated to cell-edge users rather than cell-interior users, and this way, the cell capacity is maximized. The proposed sensing mechanism can be extended for spectrum access from multiple secondary cells, the investigation of which is part of our future work.

\section{APPENDIX A \\ PROOF OF THEOREM 1}

In downlink, an SNR of user $k$ and channel $x$ at cell $i$ is expressed as

$$
\operatorname{SNR}_{k, i}(x)=\frac{p_{x} d_{k, i}^{-\alpha} c}{N+\sum_{j \in \mathbb{N}_{i}} p_{x} d_{k, j}^{-\alpha} c}
$$

where $p_{x}$ is the transmit power, $N$ is the background noise, and $d_{k, i}$ represent the distance between user $k$ and BS $i$ (or interfering user $i$ in the neighboring cell in case of uplink). Because $N$ is negligible compared with intercell interference, we can rewrite (11) as

$$
\frac{1}{\operatorname{SNR}_{k, i}(x)}=\sum_{j \in \mathbb{N}_{i}}\left(\frac{d_{k, j}}{d_{k, i}}\right)^{-\alpha} .
$$

In general, $\left(d_{k, j} / d_{k, i}\right)>1$, because the distance between a certain user and its serving BS is less than the distance between the user and its neighboring $\mathrm{BS}^{8}{ }^{8}$ As $\alpha$ increases, $1 / \operatorname{SNR}_{k, i}(x)$ decreases, which causes the Shannon capacity to increase. Therefore, each user achieves higher capacity when the channel is more attenuated by path loss.

\section{APPENDIX B \\ PROOF OF THEOREM 2}

First, we consider case 1, where the intercell interference is greater than the background noise. Assume that channels are optimally allocated to users and two users 1 and 2 in cell $i\left(d_{1, i}>d_{2, i}\right)$ are assigned to $f_{o}$ and $f_{s}$, respectively, each with a path-loss exponent $\alpha_{o}$ and $\alpha_{s}\left(\alpha_{o}>\alpha_{s}>1\right)$. In the following proof, downlink is only considered, because this proof also holds for uplink if neighboring BSs are replaced by MTs in neighboring cells and the network information, as shown in Table I, is distributed to MTs. We will prove it by showing that this two-user case (case $\mathrm{A}$ in the following discussion) contradicts the objective of the maximization of cell capacity. We compare the following two cases and find the case that maximizes the sum of capacities.

- Case A. $f_{o}$ is allocated to user 1 , and $f_{s}$ is allocated to user 2, yielding $C_{1, o}+C_{2, s}$.

- Case B. $f_{s}$ is allocated to user 1 , and $f_{o}$ is allocated to user 2, yielding $C_{1, s}+C_{2, o}$.

This condition is equivalent to comparing $C_{1, o}-C_{1, s}$ and $C_{2, o}-C_{2, s}$. Because each capacity is determined by the SNR as in (12), we can compare

$$
\frac{1+1 / \sum_{j \in \mathbb{N}_{i}}\left(\frac{d_{1, j}}{d_{1, i}}\right)^{-\alpha_{o}}}{1+1 / \sum_{j \in \mathbb{N}_{i}}\left(\frac{d_{1, j}}{d_{1, i}}\right)^{-\alpha_{s}}} \text { and } \frac{1+1 / \sum_{j \in \mathbb{N}_{i}}\left(\frac{d_{2, j}}{d_{2, i}}\right)^{-\alpha_{o}}}{1+1 / \sum_{j \in \mathbb{N}_{i}}\left(\frac{d_{2, j}}{d_{2, i}}\right)^{-\alpha_{s}}} .
$$

For simplicity, we assume that two users see the same neighboring BSs, which dominantly interfere, and hereafter, $\mathbb{N}$ only includes such dominant neighboring BSs. The distance from neighboring cells that dominantly interfere becomes shorter as the distance from the serving BS becomes longer. Because $d_{1, i}>d_{2, i}$, we obtain $d_{1, j}<d_{2, j}$ for such a neighboring BS $j$. Therefore, $d_{1, j} / d_{1, i}<d_{2, j} / d_{2, i}$. Representing these ratios of distances as $y_{j} \triangleq d_{k, j} / d_{k, i}$, we can generalize each term in (13) as

$$
g\left(y_{1}, \cdots\right)=\frac{1+1 / \sum_{j \in \mathbb{N}_{i}} y_{j}^{-\alpha_{o}}}{1+1 / \sum_{j \in \mathbb{N}_{i}} y_{j}^{-\alpha_{s}}} .
$$

Because $\alpha_{o}>\alpha_{s}$, the denominator more quickly increases than the numerator when all $y_{j}$ 's increase. When we choose the biggest $y_{j}$ in (14), i.e., BS $j$, which dominantly interferes, we can simply conclude that the latter term in (13) is higher, which means that case B will achieve a higher sum of capacities.

\footnotetext{
${ }^{8}$ Some cell-edge users, before carrying out a handoff, may experience $\left(d_{k, j} / d_{k, i}\right)<1$. In this case, the user may achieve higher capacity when accessing a TV channel rather than a licensed channel, which is contrary to this theorem. However, accessing a TV channel for such users still contributes to the maximization of cell capacity, which is consistent with Theorem 2.
} 
Therefore, a low carrier frequency should be allocated to users who are located farther from the BS to maximize the cell capacity.

For case 2, where the background noise is greater than the intercell interference, (11) is simply written as follows:

$$
\operatorname{SNR}_{k, i}(x)=\frac{p_{x} d_{k, i}^{-\alpha} c}{N} .
$$

By applying the aforementioned two-user case, we can similarly prove that case B achieves a higher sum of capacities.

\section{APPENDIX C \\ PROOF OF THEOREM 3}

We compare the following two scenarios: 1) in WRAN systems, a WRAN BS interferes and 2) in our system model, $n$ partially PU-free BSs interfere with a potential PU. Assume that a PU exists at the same location with distance $r$ from the cell boundary of WRAN and our systems. The distance between each BS and the PU is approximately $R_{\text {cell }}+r$ and $R_{\text {wran }}+r$ in each scenario. Then, we want to prove that the aggregated interference from the partially PU-free BSs in the worst case $n p_{s}\left(R_{\text {cell }}+r\right)^{-\alpha_{s}} c$ is less than the interference from the WRAN BS, i.e., $n p_{s}\left(R_{\text {cell }}+r\right)^{-\alpha_{s}} c<p_{\text {wran }}\left(R_{\text {wran }}+\right.$ $r)^{-\alpha_{s}} c$. Based on (10), we derive

$$
\begin{aligned}
\frac{n p_{s}\left(R_{\text {cell }}+r\right)^{-\alpha_{s}}}{p_{\text {wran }}\left(R_{\text {wran }}+r\right)^{-\alpha_{s}}} & \leq\left(\frac{R_{\text {wran }}}{R_{\text {cell }}}\right)^{-\alpha_{s}}\left(\frac{R_{\text {cell }}+r}{R_{\text {wran }}+r}\right)^{-\alpha_{s}} \\
& =\left(\frac{1+r / R_{\text {cell }}}{1+r / R_{\text {wran }}}\right)^{-\alpha_{s}}<1
\end{aligned}
$$

where the last inequality holds, because $R_{\text {cell }}<R_{\text {wran }}$.

\section{REFERENCES}

[1] A. Hashimoto, H. Yoshino, and H. Atarashi, "Roadmap of IMT-Advanced development," IEEE Microw. Mag., vol. 9, no. 4, pp. 80-88, Aug. 2008.

[2] Award of Spectrum in the Bands $800 \mathrm{MHz}, 1.8 \mathrm{GHz}, 2.0 \mathrm{GHz}$ and $2.6 \mathrm{GHz}$ for Wireless Access for the Provision of Telecommunications Services, Reference: BK 1a-09/002, Oct. 12, 2009.

[3] J. Mitola, III and G. Q. Maguire, Jr., "Cognitive radio: Making software radios more personal," IEEE Pers. Commun., vol. 6, no. 4, pp. 13-18, Aug. 1999.

[4] Fed. Commun. Comm., Second memorandum opinion and order (FCC 10-174), Sep. 2010. [Online]. Available: http://transition.fcc.gov/Daily_ Releases/Daily_Business/2010/db0923/FCC-10-174A1.pdf

[5] IEEE 802.22 Working Group on Wireless Regional Area Networks. [Online]. Available: http://www.ieee802.org/22/

[6] C. Cordeire, K. Challapali, D. Birru, and S. Shanker N, "IEEE 802.22: An introduction to the first wireless standard based on cognitive radios," J. Commun., vol. 1, no. 1, pp. 38-47, Apr. 2006.

[7] T. Weiss and F. K. Jondral, "Spectrum pooling: An innovative strategy for the enhancement of spectrum efficiency," IEEE Commun. Mag., vol. 42, no. 3, pp. 8-14, Mar. 2004.

[8] S. Haykin, "Cognitive radio: Brain-empowered wireless communications," IEEE J. Sel. Areas Commun., vol. 23, no. 2, pp. 201-220, Feb. 2005.

[9] I. F. Akyildiz, W.-Y. Lee, M. C. Vuran, and S. Mohanty, "NeXt generation/dynamic spectrum access/cognitive radio wireless networks: A survey," Comput. Netw., vol. 50, no. 13, pp. 2127-2159, Sep. 2006.

[10] R. Urgaonkar and M. J. Neely, "Opportunistic scheduling with reliability guarantees in cognitive radio networks," in Proc. IEEE INFOCOM, 2008, pp. 1301-1309.
[11] S. Huang, X. Liu, and Z. Ding, "Opportunistic spectrum access in cognitive radio networks," in Proc. IEEE INFOCOM, 2008, pp. 1427-1435.

[12] B. Ishibashi, N. Bouabdallah, and N. Bouabdallah, "QoS performance analysis of cognitive radio-based virtual wireless networks," in Proc. IEEE INFOCOM, 2008, pp. 2423-2431.

[13] V. Stavroulaki, S. Buljore, P. Roux, and E. Melin, "Equipment management issues in $\mathrm{B} 3 \mathrm{G}$, end-to-end reconfigurable systems," IEEE Wireless Commun., vol. 13, no. 3, pp. 24-32, Jun. 2006.

[14] Z. Boufidis, R. Falk, N. Alonistioti, E. Mohyeldin, N. Olaziregi, and B. Souville, "Network support modeling, architecture, and security considerations for composite reconfigurable environments," IEEE Wireless Commun., vol. 13, no. 3, pp. 34-44, Jun. 2006.

[15] D. Cabric, S. M. Mishra, and R. W. Brodersen, "Implementation issues in spectrum sensing for cognitive radios," in Proc. Asilomar Conf. Signals, Syst., Comput., Nov. 2004, pp. 772-776.

[16] H. Tang, "Some physical-layer issues of wideband cognitive radio systems," in Proc. IEEE DySPAN, Nov. 2005, pp. 151-159.

[17] H. Kim and K. G. Shin, "In-band spectrum sensing in cognitive radio networks: Energy detection or feature detection?," in Proc. ACM MOBICOM, Sep. 2008, pp. 14-25.

[18] C. Cordeiro, M. Ghosh, D. Cavalcanti, and K. Challapali, "Spectrum sensing for dynamic spectrum access of TV bands," in Proc. CrownCom, 2007, pp. 225-233.

[19] Y.-C. Liang, Y. Zeng, E. C. Y. Peh, and A. T. Hoang, "Sensing-throughput tradeoff for cognitive radio networks," IEEE Trans. Wireless Commun., vol. 7, no. 4, pp. 1326-1337, Apr. 2008.

[20] Y. Selen, H. Tullberg, and J. Kronander, "Sensor selection for cooperative spectrum sensing," in Proc. IEEE DySPAN, 2008, pp. 1-11.

[21] Y. Yuan, P. Bahl, R. Chandra, T. Moscibroda, and Y. Wu, "Allocating dynamic time-spectrum blocks in cognitive radio networks," in Proc. ACM MobiHoc, 2007, pp. 130-139.

[22] K. Xing, X. Cheng, L. Ma, and Q. Liang, "Superimposed code-based channel assignment in multiradio multichannel wireless mesh networks," in Proc. ACM MobiCom, 2007, pp. 15-26.

[23] J.-A. Bazerque and G. B. Giannakis, "Distributed scheduling and resource allocation for cognitive OFDMA radios," in Proc. CrownCom, 2007, pp. 343-350.

[24] H.-S. Chen, W. Gao, and D. G. Daut, "Spectrum sensing for wireless microphone signals," in Proc. IEEE SECON, San Francisco, CA, Jun. 2008, pp. 1-5.

[25] Z. Lei and F. Chin, "A reliable and power-efficient beacon structure for cognitive radio systems," IEEE Trans. Broadcast., vol. 54, no. 2, pp. 182 187, Jun. 2008.

[26] Y. Selen and J. Kronander, "Cooperative detection of programme making special event devices in realistic fading environments," in Proc. DySPAN, Singapore, Apr. 2010, pp. 1-9.

[27] C. Cordeiro and K. Challapali, "C-MAC: A cognitive MAC protocol for multichannel wireless networks," in Proc. DySpan, Apr. 2007, pp. 147-157.

[28] Qualcomm Europe, 3GPP TSG-RAN WG1 \# 55 Coordinated Multipoint downlink transmission in LTE-Advanced, Nov. 2008.

[29] Y.-J. Choi, Y. Xin, and S. Rangarajan, "Overhead-throughput tradeoff in cooperative cognitive radio networks," in Proc. IEEE WCNC, 2009, pp. 1-6.

[30] M. Hata, "Empirical formula for propagation loss in land mobile radio services," IEEE Trans. Veh. Technol., vol. VT-29, no. 3, pp. 317-325, Aug. 1980 .

[31] H. K. Chung and H. L. Bertoni, "Range-dependent path-loss model in residential areas for the VHF and UHF bands," IEEE Trans. Antennas Propag., vol. 50, no. 1, pp. 1-11, Jan. 2002.

[32] T. R. Rao, S. V. B. Rao, M. V. S. N. Prasad, M. Sain, A. Iqbal, and D. R. Lakshmi, "Mobile radio propagation path loss studies at VHF/UHF bands in Southern India," IEEE Trans. Broadcast., vol. 46, no. 2, pp. 158 164, Jun. 2000

[33] C. S. Leem, S. W. Kim, C.-J. Kim, S.-C. Kang, and J. Lee, "Spectral efficiency of WRAN spectrum overlay in the TV white space," ETRI J., vol. 30, no. 6, pp. 871-873, Dec. 2008

[34] Fed. Commun. Comm., ET Docket no. 03-122 Revision of Parts 2 and 15 of the Commission's Rules to Permit Unlicensed National Information Infrastructure (U-NII) Devices in the 5-GHz Band, Nov. 18, 2003.

[35] S.-E. Elayoubi, O. Ben Haddada, and B. Fourestie, "Performance evaluation of frequency planning schemes in OFDMA-based networks," IEEE Trans. Wireless Commun., vol. 7, no. 5, pp. 1623-1633, May 2008.

[36] Qualcomm R1-050896, 3GPP TSG-RAN WG1 \#42 Description and Simulations of Interference Management Technique for OFDMA-Based E-UTRA Downlink Evaluation, Aug. 2005. 


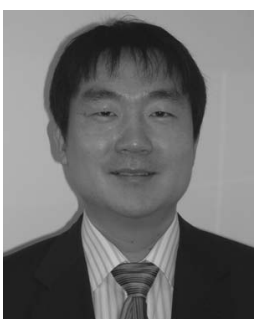

Young-June Choi (M'08) received the B.S., M.S., and Ph.D. degrees from Seoul National University, Seoul, Korea, in 2000, 2002, and 2006, respectively.

From September 2006 to July 2007, he was a Postdoctoral Researcher with the University of Michigan, Ann Arbor. From 2007 to 2009, he was with NEC Laboratories America, Princeton, NJ, as a Research Staff Member. He is currently an Assistant Professor with Ajou University, Suwon, Korea. His research interests include fourth-generation wireless networks, radio resource management, and cognitive radio

networks.

Dr. Choi is the recipient of the Gold Prize at the Samsung Humantech Thesis Contest in 2006 .

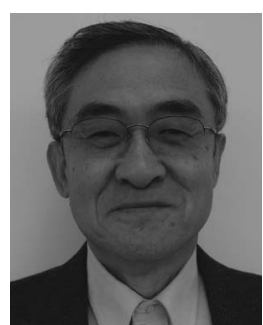

Kang G. Shin (LF'12) received the B.S. degree in electronics engineering from Seoul National University, Seoul, Korea, in 1970 and the M.S. and Ph.D. degrees in electrical engineering from Cornell University, Ithaca, NY, in 1976 and 1978, respectively.

From 1978 to 1982 , he was with the faculty of Rensselaer Polytechnic Institute, Troy, NY. He also chaired the Computer Science and Engineering Division, Department of Electrical Engineering and Computer Science, The University of Michigan Ann Arbor, for three years beginning January 1991. He is currently the Kevin and Nancy O'Connor Professor of Computer Science with the Department of Electrical Engineering and Computer Science, University of Michigan, Ann Arbor. He has supervised the completion of 69 Ph.D. dissertations and is the author or a coauthor of about 760 technical articles and one textbook. He is the holder of more than 20 patents or invention disclosures. His research interests include computing systems and networks, as well as embedded real-time and cyber-physical systems, with emphasis on timeliness, security, and dependability.

Prof. Shin is a Fellow of the Association for Computing Machinery (ACM) and has served on many editorial boards, including the IEEE TRANSACTIONS on PARALlEL AND DistRIBUTED SySteMS and the ACM Transactions on Embedded Systems. He has been the Chair of several major conferences, including the 15th ACM International Conference on Mobile Computing and Networking in 2009, the Fifth IEEE Communications Society Conference on Sensor, Mesh, and Ad Hoc Communications and Networks in 2008, the Third ACM/USENIX International Conference on Mobile Systems, Applications, and Services in 2005, the Sixth IEEE Real-Time Technology and Applications Symposium in 2000, and the Eighth IEEE Real-Time Systems Symposium in 1987. He has also served on numerous government committees such as the U.S. National Science Foundation Cyber-Physical Systems Executive Committee and the Korean Government R\&D Strategy Advisory Committee. $\mathrm{He}$ has also received numerous best paper awards, including the Best Paper Award at the 2011 IEEE International Conference on Autonomic Computing and the 2000 and 2010 USENIX Annual Technical Conferences, the 2003 William R. Bennett Prize Paper Award from the IEEE Communications Society, and the 1987 Outstanding IEEE TRANSACTIONS OF Automatic ControL Paper Award. He has also received several institutional awards, including the Research Excellence Award in 1989, the Outstanding Achievement Award in 1999, the Distinguished Faculty Achievement Award in 2001, and the Stephen Attwood Award in 2004 from the University of Michigan. He received the Distinguished Alumni Award from the College of Engineering, Seoul National University, in 2002, the 2003 IEEE RTC Technical Achievement Award, and the 2006 Ho-Am Prize in Engineering. 